\title{
Inscribing Gender: A Duoethnographic Examination of Gendered Values and Practices in Fitness Tracker Design
}

\author{
Marika Cifor \\ Indiana University Bloomington \\ Bloomington, United States \\ mcifor@iu.edu
}

\author{
Patricia Garcia \\ University of Michigan \\ Ann Arbor, United States \\ garciapg@umich.edu
}

\begin{abstract}
Using fitness trackers to generate and collect quantifiable data is a widespread practice aimed at better understanding one's health and body. The intentional design of fitness trackers as genderless or universal is predicated on masculinist design values and assumptions and does not result in "neutral" artifacts. Instead, $\backslash$ ignoring gender in the design of fitness tracking devices marks a dangerous ongoing inattention to the needs, desires, lives, and life chances of women, as well as transgender and gender nonconforming persons. We utilize duoethnography, a methodology emphasizing personal narrative and dialogue, as a tool that promotes feminist reflexivity in the design and study of fitness tracking technologies. Using the Jawbone UP3 as our object of study, we present findings that illustrate the gendered physical and interface design features and discuss how these features reproduce narrow understandings of gender, health, and lived experiences.
\end{abstract}

\section{Introduction}

Fitness trackers have become a ubiquitous technology, dominating a rapidly growing wearables market. The tremendous scale of the market and the quotidian incorporation of such fitness tracking devices into daily life reflects a "measuring mania," where knowledge of one's gendered body and its health is produced through seemingly objective, universal, and quantifiable representations such as daily steps or sleep duration. Lupton [35] argues that the "lure of numbers" through the widespread phenomenon of digital health tracking is producing an "algorithmic subjectivity" that shapes and normalizes users' understandings of gender, bodies, and behaviors; she describes these devices as "disciplinary" in their shaping of gendered bodies in ways that render them available and "amenable" to monitoring and tracking. Additionally, as Bowker and
Star [5] have powerfully argued, any technology of measurement and classification serves to legitimate particular forms of knowledge and experience, while rendering others invisible and illegible.

We argue that fitness tracking devices perpetuate a masculinist ideal of an adherent universal, genderless healthy user. Rather than resulting in "neutral" artifacts, the aim to create genderless devices paradoxically highlights the device's inability to account for gendered health concerns. Additionally, when gender is addressed in design decisions, it promotes highly normative conceptions of women and of femininity. In this paper, we identify a series of gendered implications in the physical and interface design of the Jawbone UP3 and its associated app. We assert that fitness tracking design tacitly supports regimes of disciplinary practices devoted to the attainment of normative femininity, while at the same time perpetuating dangerous inattention to the real needs, desires, bodies, lives, and life chances of women, transgender, and gender non-conforming persons.

Drawing on a six-month study of fitness tracking with the Jawbone UP3, we utilize duoethnography as a feminist methodology to uncover "common pain points and overlooked opportunities" unique to gendered experiences and interactions with sociotechnical systems [13]. First, we contextualize the relations of gender and fitness tracking within scholarship on digital health tracking and on feminist approaches to socio-technical systems design and research. Second, we outline our research methodology and establish the utility of duoethnography as a tool for promoting feminist reflexivity in the design and study of fitness tracking technologies. Finally, we present findings that illustrate the gendered physical and interface design features and discuss how these features reproduce narrow understandings of gender, health, and lived experiences. 


\section{Background}

\subsection{Digital health tracking}

Fitness trackers have become a ubiquitous technology. One in ten adults in the United States already uses a fitness tracker. Projections estimate that that number is likely to continue to increase rapidly $[40,47]$. The popularity of these devices provides consumers with numerous avenues for undertaking the process of self-tracking, defined as the practice of collecting, recording, and analyzing personal data to produce statistics that describe one's habits, behaviors, and feelings [35]. Self-tracking is typically utilized as a means to produce self-knowledge through the process of collecting data on a diverse set of measurable physiological, behavioral, and environmental conditions, including diet, mood, sleep, and health triggers such as air quality. Personal data collection related to health and wellness has risen in popularity [20] and become a widespread activity due to social and economic factors including increasing emphasis on personalized health management [50], the growing wearables market, and advancements in sensing technologies [12]. For instance, companies such as Nike, FitBit, and Garmin have all created popular consumer wearables capable of capturing physiological data points such as blood pressure, skin conductance, movement, sleep patterns, and heart rate [16]. In addition to technological advances, the use of mobile devices such as phones, tablets and smartwatches have made digital health tracking more accessible via the development of user-friendly apps that visually represent health data in colorful graphs and pie charts. Thus, advances in sensing technologies coupled with the development of mobile health (mHealth) applications have resulted in the widespread adoption of digital health tracking among American consumers. As of 2015, a majority of fitness tracker users were women (54 percent) [40] and market research reveals gender-based variance in self-tracking practices [47]. Yet, gender has not received due attention from designers or researchers working on this area [17].

Despite the rising popularity of digital health tracking, research has identified several pressing issues, including technology abandonment [12], privacy concerns over third-party access to users' health data [43], accuracy of data collection [11], workplace surveillance via health programs [22], and the commodification of personal data [51]. In addition to these issues, we argue that understanding one's body through universalized commensurable data points flattens the depth and complexity of gendered personal health and life experiences by relegating them to measurable physiological parameters and symptoms.
As a result, the simplification of health experiences contributes to a masculinist normalization that positions ideal citizens as those that take selfresponsibility $[34,47]$ and privileges healthist views of the self that overlook gender and other cultural and economic determinants of health and wellness.

Women have long been encouraged within our patriarchal society to adopt practices and behaviors that produce bodies that adhere to norms of feminine beauty [47,54]. Feminist scholars have posited that digital self-tracking "may engage women in ever more intense, intricate, and extensive regimes of selfdiscipline and self-perfection" [47:50]. Digital health tracking is produced by and reproduces highly limited conceptualizations of health and wellness dependent on quantification and gendered social norms. Ultimately, the design of these trackers obscures the importance of gendered embodiments and alternative forms of selfknowledge.

\subsection{Feminist studies of socio-technical systems}

In response to the flattening of gender and gendered health experiences, we argue that there is a need to critically examine the design of digital health tracking devices and the data they produce in ways that counter normalization and universality. Feminist approaches to studying sociotechnical systems offer a set of theories and concepts that facilitate critical and pluralistic engagements with issues of power, dominance, and privilege. Feminist HCI scholarship draws from related work in feminist science and technology studies that utilizes feminist theory to make interventions in technology research. For example, scholars have employed feminist standpoint theory [25] and theorizations of gender performativity [10] to counter traditional notions of objectivity, to demonstrate the value of marginalized positionalities in the field $[8,14,27]$, and to address the need for critical reflexivity and attention to complex understandings of gender in digital and social media [44,53]. Feminist study of sociotechnical systems can be situated within a longer intellectual genealogy of critical work that has included the challenging of established identity models [21,24,26,32], defamiliarization of domestic technologies [3], adoption and appropriation of gender in IT [23], and the operationalization of intimacy and sexuality in HCI [31]. In general, these studies promote interrogation of power, gender, and inequity through reflexivity and the centering the socio-technical needs, desires, values and experiences of diverse users.

In our work, we build upon feminist research that has examined devices as sociotechnical design objects $[1,13,14,18,53]$ and scholarship that has specifically focused on women's health experiences such as those 
related to body disruption and intimate care [1], and menstruation [17]. Our study makes what Bardzell has outlined as a critique-based contribution by "analyz[ing] designs...to expose their unintended consequences" [2:1301] and by reading technological devices for the "tacit assumptions" they make and "latent cultural values" they express about women and femininity [54:372-373]. By focusing specifically on the experiences of women, our work aligns with feminist research that questions Western universalism [13] and aims to move away from "gender-agnostic" [15] views of technology and data. Digital health technologies, in particular, capture highly personal data that is inextricably linked to contextual factors and one's complex identity, which includes intersections of gender as well as race, sexuality, class, and ability.

\section{Methodology}

\subsection{Duoethnography}

Duoethnography is a qualitative, iterative approach for studying how two or more researchers experience and give different meaning to a shared phenomenon. This methodology employs personal narrative to simultaneously generate, interpret, and articulate data about a common phenomenon [39]. We position the methodology as a feminist tool due to its emphasis on critically engaging with the subjective and affective as valid sites of knowledge production, as well as its call for establishing relational ethics practices. We present duoethnography as a generative tool that can promote alternative ways of knowing and meaning making [29] around concepts such gender, health, and wellness by capturing subjective interactions between users, devices, and data. Thus, we employ duoethnography as an emerging feminist methodology that centers users' gendered experiences, embodiments, and identities to offer an explicitly feminist accounting of the present and potential roles of self-tracking in regulating women's bodies and subjectivities and reinforcing gender-based inequalities. Prior duoethnographic work has largely confined to fields such as education $[7,48]$, communication [30], and critical ethnic studies [29]. The majority of this research has been conducted by teams of two or three co-researchers who engage in direct in-person dialogues, formally or informally, over a set period of time around a particular predetermined subject or a set of subjects. These dialogues take various forms, ranging from structured discussions guided by a predetermined set of structured questions to conversational shared probings of a topic or data point. Dialogue between co-researchers distinguishes duoethnography from other ethnographic approaches and can involve a range of dialectical interactions, including personal narrative and reactions to cultural and historical artifacts [49]. Drawing upon existing literature on duoethnography, we have developed four descriptive facets of duoethnography (table 1).

\section{Table 1. Four facets of duoethnography}

\begin{tabular}{|l|l|}
\hline \multicolumn{1}{|c|}{ Facet } & \multicolumn{1}{|c|}{ Description } \\
\hline Relationality & $\begin{array}{l}\text { Centering multiple and complex } \\
\text { connections among individual } \\
\text { researchers, participants, future readers, } \\
\text { technologies, and the self }\end{array}$ \\
\hline Difference & $\begin{array}{l}\text { Providing space for a multiplicity of } \\
\text { perspectives, values, and disparate ways } \\
\text { of knowing }\end{array}$ \\
\hline $\begin{array}{l}\text { Dialogic } \\
\text { Process }\end{array}$ & $\begin{array}{l}\text { Practicing engaged and interactive } \\
\text { dialogue as a primary approach for the } \\
\text { shared probing of a theme, activity, } \\
\text { event, or problem }\end{array}$ \\
\hline $\begin{array}{l}\text { Critical } \\
\text { Subjectivity }\end{array}$ & $\begin{array}{l}\text { Interrogating individual and collective } \\
\text { feelings, experiences, values, or } \\
\text { perspectives as a vital component of the } \\
\text { research process }\end{array}$ \\
\hline
\end{tabular}

We utilized these facets to iteratively investigate digital fitness tracking, specifically the Jawbone UP3, as gendered objects of sociotechnical design embedded in political, social, cultural, and economic contexts. The Jawbone UP3 was selected based upon its market popularity, ease-of-use, relative affordability, and most importantly, its similarity in technical specifications to other trackers on the market, which allowed us to focus on popular features such as accelerometers, bioimpedance sensors, and haptic signals.

\subsection{Data Collection}

3.2.1. Diary study. In order to promote dialogue, incorporate personal narrative, and follow the practice of using the self as the site of research, the authors participated in a collaborative six-month (May through October 2017) diary study using the Jawbone UP3. The authors wore the devices daily and nightly and engaged in self-documentation via weekly diary entries. Given that acts of health tracking occur throughout the course of one's day, we decided to use online diaries to capture everyday lived experiences. Prior work has used journals and diaries as means for collecting qualitative data on people's daily experiences interfacing with technologies [28]. We iteratively developed of semistructured prompts to guide our selfdocumentation process. Each weekly entry consisted of 
responses to a predetermined topic and six recurring questions. For example, week 11 focused on "gendered experiences" and included the following question: Has the device provided advice or other feedback that you perceive as gendered? If so, what and why? Topical questions allowed us to critically address the experiences of digital health tracking and user interactions with the device from our own positionalities, such as physically wearing a tracker, inputting identity markers such as gender, publicly sharing data, and reaching and failing to attain fitness goals.

\subsection{Data analysis}

The data analysis was guided by two areas of interest: 1) to identify how gender and gendered values and worldviews were reflected in and constructed by the Jawbone UP3, and 2) to elucidate how gender shaped our shared and individual experiences related to health tracking. As dialogue is central to the duoethnographic process, we reviewed each other's diaries and conducted thematic analysis [6] through a process of verbal consensus-building. After initial themes were identified, we created codes using the data and existing literature on duoethnography, feminist HCI, and personal health informatics. The codes included: "Genderless Universal User," "Gender and Labor," "Gender in Design," "Gender Roles and Binary," "Gender and Privacy," and "Women's Health." These codes were applied to the entire corpus (44 diary entries) through an iterative coding process using Dedoose, a qualitative analysis software.

\section{Findings}

\subsection{Inscribing gender through physical design}

Feminist research has examined how gender is inscribed into technological artifacts [4] and how these inscriptions "invite or inhibit specific performances of gender identities and relations" among users [41:473]. For example, when examining Philips electric shavers, Oudshoorn, et al [41] demonstrated how gender is imprinted into the physical design of the Philishave and Ladyshave, two shavers meant to accomplish the same personal grooming task. A comparison of the two shavers revealed differences in shape (sharp angles vs. rounded), color (dark vs. pastel colors), and construction (visible vs. hidden screws). Our analysis of the tracker's physical design revealed similar gendered contrasts between the femininity of Jawbone UP3 and other popular trackers on the market such as the more stereotypically masculine Garmin vívoactive 3 .
4.1.1. Aesthetics. During the unboxing process, both authors noted that there is "something "feminine", about the design of the Jawbone UP3 band. Author 2 noted that "It is meant to look more like a bracelet or accessory" with its "small, thin," and "colorful" design. The device fits into the model of "chic" wearable tech proposed by Wissinger that is developed to market to "the fashion consumer, presumably female, healthy, and living within current feminine norms" [54:1]. The look of the Jawbone UP3 is in stark contrast to a number of other such trackers on the market, many of which favor a bulkier, bigger, black or metallic design.

The gendered aesthetics of fitness tracking were an issue particularly in the early weeks of the project for Author 1. She noted that the style and overall look of the device was a "pleasant surprise" since one of her "big hesitations has been how it will look" both from an aesthetic perspective, as well as what its physical design signaled to observers. The aesthetic and physical elements, such as color (white and gold) and size (small, thin), resulted in the device feeling and looking like an accessory. Author 1 documented an exchange with a friend who assumed the device was "a vintage bracelet at first glance." Since the device looked and felt largely like any other piece of jewelry, it blended into outfits, rather than announcing to all that she was engaged in a fitness tracking process, something with which she had marked discomfort.

Daily wear shifted Author 1's awareness of the device and self-consciousness about the design aesthetics. She noted a month in, "I've stopped thinking about whether it will match outfits." By the end of data collection, she reflected that wearing the device had "gotten to be as natural as wearing my wedding rings, something that also felt strange." The "naturalness" of wearing the tracker resulted from gendered aesthetic and physical design decisions that allowed the device to blend in seamlessly with women's fashion and accessories.

For both Author 1 and Author 2, the slim physical design contributed to their continued use of the device because it allowed for greater privacy. Author 2 felt self-conscious wearing the device. She wrote in her diary, "I feel like fitness devices are such outward signals of "health" like wearing Lululemon or crossfit t-shirts. Like "'Look at me! I work out!", The lowprofile discreet design of the fitness tracker (curved, muted colors, slim) allowed for the act of fitness tracking to remain less visible. Despite both authors favorable responses to the "feminine" aesthetics of the Jawbone UP3, it is important to note that these choices reflecting gendered assumptions about what women want or consider feminine, a thinner strap, smaller face, more subtle styling, and colorways that include 
white, gold, and other colors associated with femininity, subscribe to and reproduce dominant normative notions of gender.

4.1.2. Fit. Fitness trackers and other wearables have been criticized for perpetuating "universal" designs that incorporate stereotypically masculine features such as wide bands, rugged materials, and large displays. For those with smaller wrists, including many women, these features result in substantial fit issues. Some fitness trackers are now marketed to female consumers based on their smaller sizing capacities. On its box, the Jawbone UP3 noted in an example of universal design strategies, that "One size fits most." From the start, Author 1 had "some anxiety about whether it will actually fit properly" on her small, narrow wrist. The ways that biometric technologies employ cisgendered white men's bodies as the default template for humanity reflecting the embodiments of most of their designers has been widely noted [36]. The gendered failures in fit we encountered in this study offer further evidence of the ongoing design bias favoring male bodies.

The device, in order to reliably automatically collect data including heart rate, steps, and sleep, must be properly and tightly positioned on the wrist. Users are instructed to wear it on their non-dominant hand. Early on in our data collection, Author 1 noticed that it had failed to record sleep data for some nights when she knew she had been wearing it. Users are encouraged to wear the device 24/7, including overnight which allows them to engage with its sleep data collection and analysis functionalities. Wearing it overnight proved uncomfortable for both authors. Author 1 wrote, "The most significant physical interaction with it is that it loosens up regularly and that I still get the poorly designed clasp caught in my clothes and hair...sometimes pulling out a few strands." The clasp opening used on the device did not account for longer more stereotypically feminine hairstyles or for the more delicate features of many articles of women's clothing.

The "universal" fit of the fitness tracker translated into a loose-fitting device with a clasp that frequently caught on long hair, clothing, and left both of us with visible scratch marks elsewear on our bodies. Yet, we continued to wear it. The discomfort of nightly wear informed the high level of frustration, Author 1 felt at its failures to record sleep data. She wondered, "Did the device slide too far up my arm? I wish it would give some kind of warning [when out of place]." After a couple of weeks, the device did notify her through the app that she "should be measuring [her] heart rate" and identified "fit" as the potential culprit for the device's inability to collect the data. Author 1 then switched wrists to wear the device on her dominant right wrist, which is slightly larger; the change was self-directed as the device itself did not offer remedies for potential fit issues. However, the fit issues continued. She later wrote, "I've had a hard time keeping the band tight enough so it sometimes misses data. I think my wrist is actually a bit too small for it...it's really not effective in terms of everyday wear."

Despite the assumed normative femininity of the consumer, the diversity of user embodiments, particularly the smaller wrists and longer hairstyles of many women were not accounted for in the standardized, universal design. The physical design of the device including its stereotypically feminine aesthetic sensibilities and its issues in maintaining proper fit for diverse bodies perpetuate masculinist design values that support dominant gender norms. The look of the device is demonstrably influenced by dominant conceptions of femininity and it perpetuates gender norms about the desires and sensibilities of women as users, while simultaneously failing to meet the needs of users with diverse physical embodiments in its universalist design paradigm.

\subsection{Inscribing gender through interface design}

Previous work has devoted attention to the prominence of stereotypically feminine attributes in the aesthetics of interface design such as being predominantly pink or the use of flower and heart images in apps designed for pregnancy and menstrual tracking $[17,45]$. In order to meaningfully interact with Jawbone UP3 and access the full set of features, users must pair the device with a smartphone application that allows them to view the data collected and analyzed. The app displays automatically collected and manually entered data sleep, activity, heart rate, mood, and food consumption data. This data is visualized in a brightly colored progress bar graphs.

While it may not be pink or floral, we found that the interface design of the Jawbone UP3 was still meaningfully gendered in ways reflective of distinctly masculinist values and worldviews. Through its design affordances the device's interface perpetuates constraining normative constructions of gender identity and roles in the form of user profiles, the Smart Coach feature, and structured relationships between users and between devices.

4.2.1. Binary Gender. "Gender" is classified from the very first interaction with the interface when users are prompted to choose a gender. The profile options for "gender" provided by the Jawbone's designers reflect and reproduce conceptions of gender as binary. Establishing a user profile in the app is a required step after syncing it with the device. Before requesting that 
a new user complete any other profile information (name, height, weight, age, fitness levels and goals), the interface requests that a user assign themselves and the device a "gender." Gender is represented in a small box on the top left of the screen which displays silhouettes of two human figures, one with a triangleshaped dress and the other without. When opened there are only two options: "male" and "female." The language of employed in this "gender" classification correlates more precisely with "sex." By examination of the anatomy of an individual's reproductive system infants are assigned as "male" and "female," terms used to describe sex. In contrast "gender" names the socio-cultural roles assigned based on a person's sex or an individual's own identification based on an internal awareness of one's identity. In addition to perpetuating a limiting binary classification, the interface then also reproduces a problematic, if common, slippage between sex and gender. In our journal entries on the unboxing process, we each made note of these gender constraints. "That it only has two options," Author 1 noted, immediately "makes the device unfriendly to gender non-conforming users. It also doesn't offer any options for trans users to indicate that aspect of their health experience." Both authors identify as cisgender women, however that did not limit our discomfort with the gender binary enforced. The implications of gender classification are not articulated to users. We setup our profiles as "female." Author 1 chose to do so as she "was keen to know if and how the device would give me gendered advice."

Additionally, the interface design visually constructed gender in meaningful ways. Author 1 uploaded an image of herself to her profile during setup, while Author 2 did not upload such an image to accompany her profile. When setting up our devices to operate as "friends," enabling and automating sharing and interaction between users and our devices, the binary gender was again enforced. In place of a usersubmitted photo, the app identified Author 2 by using an automatically generated image of a silhouette that read explicitly as male with a close-cropped hairstyle, broad chin and shoulders:

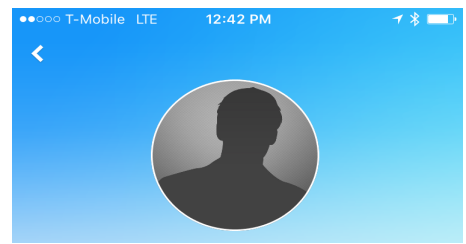

Figure 2. Default user image

This encounter made apparent that the default image for all user profiles, regardless of the gender classification assigned, is male. This visual decision reflects the worldview and perspectives of its designers, likely reflecting their own masculine identities and social roles as well as showcasing the identities of those who they assume to be using the devices. This interface design choice demonstrates how male bodies are the default body and user for digital technologies. Once we became friends, Author 2's image transformed to a different silhouette, this one a woman's head. The "female" profile image featured a hairstyle that was longer and more typically feminine with narrower shoulders and chin than its masculine counterpart. Through linguistic and visual cues Jawbone user profiles produce and reproduce dominant binary and exclusionary conceptions of gender.

4.2.2. Smart Coach Interactions. The "Smart Coach" is a central system feature of the Jawbone UP3 app. The Coach demonstrates how gender is very present through the absence of attention to it in the design of the Jawbone's software. An anthropomorphized feature that guides users in making "healthier choices" and "turns measures into meaning" (as described on the device packaging), the Smart Coach represents an attempt by designers to stimulate a particular relationship between users and devices. The notifications offered by the feature provide daily insights and encouragement aimed at empowering the user to take control of their health [50]. For Author 1, this relationship failed to feel personal or even remotely human. Yet, the relationship still reproduced gendered expectations for users' behavior and relationships with others. The Coach itself is both unnamed and ungendered, however when it is not marked the authority figure of a sports coach, reflecting the underrepresentation of women in coaching, will by default be assumed male in American culture [9,19]. Even when delivered in subtle and gentle tones, a disciplinary control through mechanisms such as the Smart Coach is exerted over women's bodies through encouraged self-compliance with convergent gendered health and beauty norms.

Based on our shared research interest in the gendered social implications of technology design and use, we included diary prompts that explicitly examine the "gendered experiences" we had with the device. Author 2 noted after reviewing the notifications from her Smart Coach for ten weeks prior that they "didn't seem to be gendered...Most of them were generic information about the importance of sleep or of not sitting for too long." We both found that the direction offered by the Smart Coach was highly repetitive and general advice on healthfulness aimed at a universal genderless user. For example, the Coach frequently offered guidance such as, consume the entire apple to "fight fat with the peel!" or, to try "going back to the playground" by jumping rope. 
However, there were a few notable moments our "female" profile assignment demonstrably informed the "personalized" advice offered. Author 1 received such a notification from the Coach on the topic of premenstrual syndrome (PMS). Under the title "Kick It," the Coach intoned, "When PMS strikes, resist the allure of the couch. A study of New Zealand women found that exercise in the days before a period lessened bloating and improved mood. Lace up and kick crabbiness to the curb."

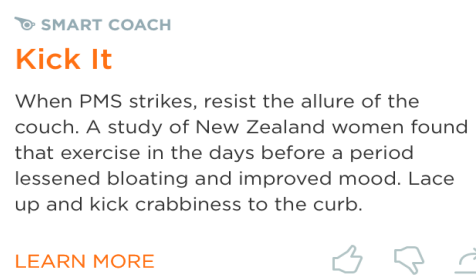

Figure 3. Personalized Smart Coach advice

Utilizing scientific studies superficially is typical of the Smart Coach's messaging. The timing of this message felt entirely random, out of sync with Author 1 's menstrual cycle. Jawbone did not sync with Clue, the menstrual tracking app she used, nor did it provide its own option for such tracking within the interface its conspicuous absence reflecting what Lupton has termed "a certain blindness to the needs of women" driven by the male-dominated nature of technology culture [33]. This PMS advice represents an almost singular pointed address of female reproductive health and wellness in the course of six months. That the unusual address of women's health in the Coach's advice was on the topic of "PMS" is significant. PMS names a set of common symptoms including bloating, headaches, and moodiness that many people who menstruate experience. While a real health condition, PMS is highly stigmatized, represented culturally primarily as the butt of jokes, as evidenced by the phrase "kick crabbiness to the curb." PMS humor reproduces assumptions about women's moodiness, irrationality, and overblown emotionality due to their embodiments and hormonal cycles [37]. Thus, even on the rare occasion when the interface did acknowledge the particularities of women's health needs it trivialized our embodied experiences.

4.2.3. Relationship Structures. The Jawbone's interface strongly encourages users to make "friends" through the app. The social interactions supported by the interface design reflect masculinized worldviews that value competition as relationship. By failing to acknowledge gender as a shaping experience, the simplistic and superficial social structures offered by the interface design failed to meet our relational needs as users. A social networking component is common among fitness trackers on the market. Networked relational components can provide users a place to share and celebrate their successes and to receive motivation when they find themselves falling short of fitness goals. Prior research has shown the ability for social processes, such as "persistent sharing" [42] to support desirable behaviors in health tracking processes. In these systems, some users serve as "persuaders" who "influence each user to "do what is best for them' [46:424]. The knowledge created by the device is envisioned by its designers not just to be greater or more precise, but to be actionable as well [50]. Therefore, in the world constructed by the Jawbone UP3 interface, our relationship was conceptualized as a source for physical action best driven through comparison. Author 2 wrote in her diary, "I don't like that this project has resulted in comparing our health stats." She noted her discomfort particularly in the push to "compare our sleep patterns" by analyzing decontextualized data such as sleep quantity, quality, and duration, which, in her view, was "then internalized as some marker of how well we're doing with our lives."

Other limited social interactions included messaging friends and allowing users to see each other's basic data, including sleep, steps, and food consumed. The possibilities for surreptitious viewing of one another's data, felt in Author 2's words "creepy" and invasive. Messaging, which reveals that you have reviewed the other person's data, did little to ameliorate our negative responses to this interface feature. The app did not ask us as new friends "about what we'd actually like to share" with one another. Reflecting on her relationship with Author 1, Author 2 wrote, "It feels weird to comment on something so personal, especially since I know Author 1 is more of a private person." The framing of relationships within the device did not consider the range of personalities and comfort of users within its structures of a reward system. The system's conception of relationships and its deployment of them for personal gain through "better" health runs counter to the personality-driven patterns of interpersonal disclosure as a means to developing and sustaining close personal relationships. "Friendship," the user-to-user and device-to-device relationship, is almost exclusively setup as a masculinist space where competition and individual gain matter most.

It is competition that the interface designers clearly see as the central motivational force for physical activity as well as centerpoint of human connection. For example, when we first initiated the relationship between our devices, the Smart Coach prompted us to engage in a "duel." While partaking in that duel, the 
next three days of our steps were compared in a "headto-head step battle" and we were sent messages pushing us to out step the other in order to claim a "victory." We agreed to participate in this competition in order to test out the feature. Author 2 hadn't actually worn the device over the course of the next few days, yet it made an explicit point of reminding her repeatedly as the "losing" user how many steps she was behind. Competition is a highly gendered social practice. Dominant norms encourage men towards competitive behavior fueled by risk taking and high levels of confidence in one's own abilities. The same gender norms discourage women from competitive behaviors, emphasizing instead values of cooperation and modesty [38,52]. There is a clear value judgment in the Jawbone's design that competition will act as a positive force, motivating users to reach their full fitness potential and to maintain high levels of interaction with the device. However, the interface design ignored gendered socialization that means competition is not understood or experienced in the same ways by many users. As person's socialized as girls we found the competition failed entirely to motivate us towards greater activity, engagement with the device, or in building a relationship with one another. As Author 2 concluded, "I don't think the comparisons are motivating. They haven't resulted in a positive competitive spirit - like "oh let me walk more!" They have actually resulted in quite the opposite."

\section{Limitations}

As an interpretivist research method, duoethnography does not aim to provide generalizable findings. Instead, in line with other forms of ethnographic research, the aim of duoethnography is to examine cultural phenomena using an inductive approach that values deeply contextualized experiences and interactions. As such, the methodology demands that researchers accurately and consistently self-report over the course of the study. Our study design mediated for the challenges of self-reporting by setting up a weekly schedule and protocol that supported the consistent collection of data via journaling, dialogues, and data log reviews. Nonetheless, we still encountered challenges in self-reporting resulting from inadvertent failures to charge the device and update its firmware and software. These gaps in data collection were addressed via supplementary journaling and dialogues that specifically reflected on the consequences of "device failures" and user mistakes.

Additionally, duoethnography is a methodology that requires trust between researchers [39]. Each researcher must be open to honestly communicating aspects of their identities and perspectives on a shared experience with each other and an audience. Sharing deeply personal experiences may be difficult and raises ethical concerns regarding privacy and disclosure in research processes. Although we began this project with an established relationship, there were moments when it felt uncomfortable to share personal information, such as our weight and diet. Our future analysis will address the discomfort of sharing personal information, further delve into tensions that arise, and explore how the affective dimensions of the project impact the interpretive process.

\section{Conclusion}

The ubiquity of fitness trackers has made them invisibly quotidian technological artifacts. The masculinist worldviews and values of designers have resulted in a purportedly genderless universal design for these trackers. However, as we have uncovered through duoethnographic study the physical and interface design features of the Jawbone UP, these devices are highly gendered. The ways that these devices and their associated practices enhance women's abilities to act as responsible citizens recording, monitoring, analyzing, and acting upon their data exerts meticulous disciplinary control over bodies and behaviors in ways that support and reproduce dominant patriarchal gender and health norms. Fitness tracking devices perpetuate constraining and limited understandings of gender, health, and diverse lived experiences, while also ignoring the real needs and particularities of the health and wellbeing of women, transgender, and gender non-conforming users. Explicit and ongoing deep interrogation by designers and researchers of the ways that these devices and their data collection, use, and dissemination practices produce and reproduce dominant socio-cultural understandings of gender, femininity, health and wellness, bodies, and subjectivities is urgently required. Inattention to gender in fitness tracking means that researchers and designers ignore the ways that these technologies might challenge entrenched stereotypes and norms.

\section{References}

1. T. Almeida, R. Comber, and M. Balaam. 2016. HCI and Intimate Care as an Agenda for Change in Women's Health. In Proceedings of the 2016 CHI Conference on Human Factors in Computing Systems (CHI '16), 2599-2611. https://doi.org/10.1145/2858036.2858187

2. S. Bardzell. 2010. Feminist HCI: Taking Stock and Outlining an Agenda for Design. In Proceedings of the SIGCHI Conference on Human Factors in Computing Systems (CHI '10), 1301-1310. https://doi.org/10.1145/1753326.1753521 
3. G. Bell, M. Blythe, and P. Sengers. 2005. Making by Making Strange: Defamiliarization and the Design of Domestic Technologies. ACM Trans. Comput.-Hum. Interact. 12, 2: 149-173. https://doi.org/10.1145/1067860.1067862

4. A.J. Berg and M. Lie. 1995. Feminism and Constructivism: Do Artifacts Have Gender? Science, Technology, \& Human Values 20, 3: 332-351. https://doi.org/10.1177/016224399502000304

5. G.C. Bowker and S.L. Star. 2000. Sorting Things Out: Classification and Its Consequences. MIT Press.

6. R.E. Boyatzis. 1998. Transforming Qualitative Information: Thematic Analysis and Code Development. SAGE.

7. R.A. Breault. 2016. Emerging issues in duoethnography. International Journal of Qualitative Studies in Education 29, 6: 777-794. https://doi.org/10.1080/09518398.2016.1162866

8. S. Breslin and B. Wadhwa. 2017. Gender and HumanComputer Interaction. In The Wiley Handbook of Human Computer Interaction. Wiley-Blackwell, 71-87. https://doi.org/10.1002/9781118976005.ch4

9. L.J. Burton. 2015. Underrepresentation of women in sport leadership: A review of research. Sport Management Review 18, 2: 155-165. https://doi.org/10.1016/j.smr.2014.02.004

10. J. Butler. 1988. Performative Acts and Gender Constitution: An Essay in Phenomenology and Feminist Theory. Theatre Journal 40, 4: 519-531.

11. M.A. Case, H.A. Burwick, K.G. Volpp, and M.S. Patel. 2015. Accuracy of Smartphone Applications and Wearable Devices for Tracking Physical Activity Data. JAMA 313, 6: 625-626. https://doi.org/10.1001/jama.2014.17841

12. J. Clawson, J.A. Pater, A.D. Miller, E.D. Mynatt, and L. Mamykina. 2015. No Longer Wearing: Investigating the Abandonment of Personal Health-tracking Technologies on Craigslist. In Proceedings of the 2015 ACM International Joint Conference on Pervasive and Ubiquitous Computing (UbiComp '15), 647-658. https://doi.org/10.1145/2750858.2807554

13. C. D'Ignazio, A. Hope, B. Michelson, R. Churchill, and E. Zuckerman. 2016. A Feminist HCI Approach to Designing Postpartum Technologies: "When I First Saw a Breast Pump I Was Wondering if It Was a Joke." In Proceedings of the 2016 CHI Conference on Human Factors in Computing Systems (CHI '16), 2612-2622. https://doi.org/10.1145/2858036.2858460

14. J.P. Dimond, C. Fiesler, and A.S. Bruckman. 2011. Domestic violence and information communication technologies. Interacting with Computers 23, 5: 413421. https://doi.org/10.1016/j.intcom.2011.04.006

15. S.M. Dray, A. Peer, A.M. Brock, A. Peters, S. Bardzell, M. Burnett, E. Churchill, E. Poole, and D.K. Busse. 2013. Exploring the Representation of Women Perspectives in Technologies. In CHI '13 Extended Abstracts on Human Factors in Computing Systems (CHI EA '13), 2447-2454. https://doi.org/10.1145/2468356.2468799

16. E. Elenko, L. Underwood, and D. Zohar. 2015. Defining digital medicine. Nature Biotechnology 33, 5: 456-461. https://doi.org/10.1038/nbt.3222
17. D.A. Epstein, N.B. Lee, J.H. Kang, E. Agapie, J. Schroeder, L.R. Pina, J. Fogarty, J.A. Kientz, and S. Munson. 2017. Examining Menstrual Tracking to Inform the Design of Personal Informatics Tools. In Proceedings of the 2017 CHI Conference on Human Factors in Computing Systems (CHI '17), 6876-6888. https://doi.org/10.1145/3025453.3025635

18. C. Fiesler, S. Morrison, and A.S. Bruckman. 2016. An Archive of Their Own: A Case Study of Feminist HCI and Values in Design. In Proceedings of the 2016 CHI Conference on Human Factors in Computing Systems (CHI '16), 2574-2585. https://doi.org/10.1145/2858036.2858409

19. L. Flanagan. 2017. The Field Where Men Still Call the Shots. The Atlantic. Retrieved from https://www.theatlantic.com/education/archive/2017/07/ the-field-where-men-still-call-the-shots/535167/

20. S. Fox and M/ Duggan. 2013. Tracking for health. Pew Research Center's Internet \& American Life Project.

21. C. Gatehouse. 2016. Feral Screens: Queering Urban Networked Publics. In Proceedings of the 2016 ACM Conference Companion Publication on Designing Interactive Systems (DIS '16 Companion), 99-104. https://doi.org/10.1145/2908805.2913014

22. N. Gorm and I. Shklovski. 2016. Steps, Choices and Moral Accounting: Observations from a Step-Counting Campaign in the Workplace. In Proceedings of the 19th ACM Conference on Computer-Supported Cooperative Work \& Social Computing (CSCW'16), 148-159. https://doi.org/10.1145/2818048.2819944

23. E. Green. 1993. Gendered Design?: Information Technology And Office Systems. CRC Press.

24. O.L. Haimson. 2017. The Social Complexities of Transgender Identity Disclosure on Social Network Sites. 280-285. https://doi.org/10.1145/3027063.3027136

25. S.G. Harding. 2004. The Feminist Standpoint Theory Reader: Intellectual and Political Controversies. Psychology Press.

26. J. Hardy and S. Lindtner. 2017. Constructing a Desiring User: Discourse, Rurality, and Design in LocationBased Social Networks. In Proceedings of the 2017 ACM Conference on Computer Supported Cooperative Work and Social Computing (CSCW '17), 13-25. https://doi.org/10.1145/2998181.2998347

27. S. Harrison, P. Sengers, and D. Tatar. 2011. Making epistemological trouble: Third-paradigm HCI as successor science. Interacting with Computers 23, 5: 385-392. https://doi.org/10.1016/j.intcom.2011.03.005

28. E. Hayashi and J. Hong. 2011. A Diary Study of Password Usage in Daily Life. In Proceedings of the SIGCHI Conference on Human Factors in Computing Systems (CHI '11), 2627-2630. https://doi.org/10.1145/1978942.1979326

29. M.F. Huckaby and M.H. Weinburgh. 2015. "Spark Like a Dialectic": Difference In-between Feminisms/Duoethnography. International Review of Qualitative Research 8, 1: 49-67. https://doi.org/10.1525/irqr.2015.8.1.49

30. G.S. Hummel and S. Toyosaki. 2015. Duoethnography as Relational Whiteness Pedagogy: Human Orientation Toward Critical Cultural Labor. International Review of 
Qualitative Research 8, 1: 27-48. https://doi.org/10.1525/irqr.2015.8.1.27

31. G. Kannabiran, J. Bardzell, and S. Bardzell. 2011. How HCI Talks About Sexuality: Discursive Strategies, Blind Spots, and Opportunities for Future Research. In Proceedings of the SIGCHI Conference on Human Factors in Computing Systems (CHI '11), 695-704. https://doi.org/10.1145/1978942.1979043

32. A. Light. 2011. HCI as heterodoxy: Technologies of identity and the queering of interaction with computers. Interacting with Computers 23, 5: 430-438. https://doi.org/10.1016/j.intcom.2011.02.002

33. D. Lupton. 2015. The cultural specificity of digital health technologies. This Sociological Life. Retrieved from

https://simplysociology.wordpress.com/2015/01/25/thecultural-specificity-of-digital-health-technologies/

34. D. Lupton. 1995. The imperative of health: Public health and the regulated body. Taylor \& Francis. Retrieved September 19, 2017 from http://www.tandfonline.com/doi/pdf/10.1080/09581599 708409082

35. D. Lupton. 2014. Self-tracking Cultures: Towards a Sociology of Personal Informatics. In Proceedings of the 26th Australian Computer-Human Interaction Conference on Designing Futures: The Future of Design (OzCHI '14), 77-86. https://doi.org/10.1145/2686612.2686623

36. S. Magnet. 2011. When Biometrics Fail: Gender, Race, and the Technology of Identity. Duke University Press, Durham.

37. S. Markens. 2016. Premenstrual Syndrome (PMS). In The Wiley Blackwell Encycopedia of Gender and Sexuality Studies.

38. M. Niederle and L.Vesterlund. 2011. Gender and Competition. Annual Review of Economics 3, 1: 601630.

39. J. Norris. 2008. Duoethnography. The SAGE encyclopedia of qualitative research methods 1: 233236.

40. NPD Connected Intelligence Group. 2015. Consumers \& Wearables. Retrieved from http://www.connectedintelligence.com/our-research/wear

41. N. Oudshoorn, A.R. Saetnan, and M. Lie. 2002. On gender and things: Reflections on an exhibition on gendered artifacts. Women's Studies International Forum 25, 4: 471-483. https://doi.org/10.1016/S02775395(02)00284-4

42. K. Park, I. Weber, M. Cha, and C. Lee. 2016. Persistent Sharing of Fitness App Status on Twitter. In Proceedings of the 19th ACM Conference on ComputerSupported Cooperative Work \& Social Computing (CSCW '16), 184-194. https://doi.org/10.1145/2818048.2819921

43. G. Paul and J. Irvine. 2014. Privacy Implications of Wearable Health Devices. In Proceedings of the 7th International Conference on Security of Information and Networks (SIN '14), 117:117-117:121. https://doi.org/10.1145/2659651.2659683

44. A. Pawar and J. Redström. 2016. Publics, Participation and the Making of Umela a Pantry. International Journal of Design 10, 1: 73-84.
45. T. Peyton, E. Poole, M. Reddy, J. Kraschnewski, and C. Chuang. 2014. "Every pregnancy is different": designing mHealth for the pregnancy ecology. In Proceedings of the 2014 conference on Designing interactive systems - DIS '14, 577-586. https://doi.org/10.1145/2598510.2598572

46. S. Purpura, V. Schwanda, K. Williams, W. Stubler, and P. Sengers. 2011. Fit4Life: The Design of a Persuasive Technology Promoting Healthy Behavior and Ideal Weight. In Proceedings of the SIGCHI Conference on Human Factors in Computing Systems (CHI '11), 423432. https://doi.org/10.1145/1978942.1979003

47. R. Sanders. 2017. Self-tracking in the Digital Era: Biopower, Patriarchy, and the New Biometric Body Projects. Body \& Society 23, 1: 36-63. https://doi.org/10.1177/1357034X16660366

48. R.D. Sawyer and T. Liggett. 2012. Shifting Positionalities: A Critical Discussion of a Duoethnographic Inquiry of a Personal Curriculum of Post/Colonialism. International Journal of Qualitative Methods 11, 5: 628-651. https://doi.org/10.1177/160940691201100507

49. R.D. Sawyer and J. Norris. 2015. Duoethnography: A Retrospective 10 Years After. International Review of Qualitative Research 8, 1: 1-4. https://doi.org/10.1525/irqr.2015.8.1.1

50. T. Sharon. 2017. Self-Tracking for Health and the Quantified Self: Re-Articulating Autonomy, Solidarity, and Authenticity in an Age of Personalized Healthcare. Philosophy \& Technology 30, 1: 93-121. https://doi.org/10.1007/s13347-016-0215-5

51. I. Shklovski, J. Vertesi, E. Troshynski, and P. Dourish. 2009. The Commodification of Location: Dynamics of Power in Location-based Systems. In Proceedings of the 11th International Conference on Ubiquitous Computing (UbiComp '09), 11-20. https://doi.org/10.1145/1620545.1620548

52. M. Sutter and D. Glätzle-Rützler. 2015. Gender Differences in the Willingness to Compete Emerge Early in Life and Persist. Management Science 61, 10: 2339-2354. https://doi.org/10.1287/mnsc.2014.1981

53. N.A. Van House. 2011. Feminist HCI meets facebook: Performativity and social networking sites. Interacting with Computers 23, 5: 422-429. https://doi.org/10.1016/j.intcom.2011.03.003

54. E. Wissinger. 2017. From "Geek" to "Chic": Wearable Technology and the Woman Question. In Digital Sociologies. Policy Press, London. 